\title{
High Plasmodium infection and multiple insecticide resistance in a major malaria vector Anopheles coluzzii from Sahel of Niger Republic
}

\author{
Sulaiman S. Ibrahim ${ }^{1,2^{*}}$, Muhammad M. Mukhtar², Helen Irving ${ }^{1}$, Rabiou Labbo ${ }^{3}$, Michael O. Kusimo ${ }^{4}$ \\ Izamné Mahamadou ${ }^{3}$ and Charles S. Wondji ${ }^{1,4^{*}}$
}

\begin{abstract}
Background: Information on insecticide resistance and the mechanisms driving it in the major malaria vectors is grossly lacking in Niger Republic, thus hindering control efforts. To facilitate evidence-based malaria control, the role of Anopheles coluzzii population from southern Niger, in malaria transmission, its insecticides resistance profile and the molecular mechanisms driving the resistance were characterized.

Methods: Blood fed female Anopheles gambiae sensu lato resting indoor were collected at Tessaoua, Niger. Source of blood was established using PCR and infection with Plasmodium determined using TaqMan assay. Resistance profile was established with the major public health insecticides, and resistance intensity determined with deltamethrin. Synergist assays were conducted with piperonyl butoxide and diethyl maleate. Presence of L1014F and L1014S knockdown resistance ( $k d r$ ) mutations in the voltage-gated sodium channel (VGSC) was investigated using TaqMan genotyping, and strength of selection pressure acting on the Anopheles populations determined by assessing the genetic diversity of a fragment spanning exon-20 of the VGSC from alive and dead females.
\end{abstract}

Results: High human blood index (96\%) and high Plasmodium falciparum infection ( 13\%) was observed in the An. coluzzii population. Also, a single mosquito was found infected with Plasmodium vivax. High pyrethroid and organochloride resistance was observed with mortalities of less than $20 \%$ for deltamethrin, permethrin, a-cypermethrin, and DDT. A high $\mathrm{LD}_{50}(156.65 \mathrm{~min})$ was obtained for deltamethrin, with a resistance ratio of $\sim 47.18$ compared to the susceptible Ngoussou colony. Moderate carbamate resistance was observed, and a full susceptibility to organophosphates recorded. Synergist bioassays with piperonyl butoxide and diethyl maleate significantly recovered deltamethrin and DDT susceptibility, respectively implicating CYP450 s (mortality $=82 \%, X^{2}=84.51, p<0.0001$ ) and glutathione S-transferases (mortality $=58 \%, x^{2}=33.96, p<0.001$ ) in resistance. A high frequency of $1014 \mathrm{~F} k d r$ mutation (82\%) was established, with significant difference in genotype distribution associated with permethrin resistance [odds ratio $=7.71\left(95 \% \mathrm{Cl} 2.43-14.53, x^{2}=13.67, \mathrm{p}=0.001\right.$ ]. Sequencing of intron-1 of the voltage-gated sodium channel (VGSC) revealed a low genetic diversity.

Conclusion: High pyrethroid resistance highlight the challenges to the effectiveness of the pyrethroids-based ITNs and indoor residual spraying (IRS) against An. coluzzii in Niger. The pyrethroids-synergists LLINs and organophosphatebased IRS maybe the alternatives for malaria control in southern Niger.

Keywords: Plasmodium falciparum, An. coluzzii, Malaria, Sahel, Insecticides, Metabolic, Resistance, kdr

\footnotetext{
*Correspondence: SulaimanSadi.lbrahim@lstmed.ac.uk;

Charles.Wondji@lstmed.ac.uk

1 Vector Biology Department, Liverpool School of Tropical Medicine

(LSTM), Pembroke Place L3 5QA, UK

Full list of author information is available at the end of the article
} 


\section{Background}

The global fight against malaria is at a crossroads with the progress made for almost two decades stalled [1, 2]. The decline in malaria burden between 2000 and 2015 [3] is facing serious setbacks, as cases of malaria have consistently increased globally between 2015 and 2017 [1, 4]. This malaria transmission recurrence, threatening a return to the 2012 levels is a warning sign to the control and elimination efforts of the World Health Organization (WHO) [5]. The trend suggests that primary regions of interests for pre-elimination need urgent attention; most worrying being the WHO African Region, which accounts for $\sim 92 \%$ of all malaria-related deaths [2]. Niger Republic, with a high transmission and increased case incidence (2010-2016), is among countries with the highest per capita rate of malaria fatalities globally [6]. It alone accounts for $\sim 4 \%$ of malaria burden in the world [4]. The Sahelian region of Niger represents the northern limit of malaria endemicity, compared to the north of the country where malaria is more marginal, where population densities are lower, and Anopheles densities are known to be very low [7]. The Sahel Region, characterized by a high seasonal malaria transmission [8], is important for monitoring purposes, to provide enough evidences to support elimination in West Africa. However, generating reliable data on indigenous Sahelian Anopheles species, their contribution to malaria transmission and monitoring insecticide resistance status over time and space [4], is a pre-requisite for implementation of evidence-based control measures in this dry region.

The major vectors of malaria in Niger are Anopheles gambiae sensu lato (s.l.) and Anopheles funestus [4, 9, 10], with Anopheles coluzzii being the dominant species from the Gambiae complex [11]. Unfortunately, information on the insecticide resistance status of these dominant vector species (DVS) from Niger and the underlying molecular mechanisms driving the resistance in the field is very limited. In addition, there is also a dearth of information on the impact of nationwide distribution of long-lasting insecticidal bed nets on development and/or escalation of resistance. Between 2005 and 2009 more than 6 million LLINs (Permanet ${ }^{\circledR} 2.0$, Vestergaard, Lausanne, Switzerland) were freely distributed, with 73.4\% coverage. Additionally, 11.3 million nets were distributed between 2014 and 2017 [12]. A study published in 2017 described pyrethroids and DDT resistance in An. coluzzii from Niger [9]. However, collection for the study was carried out in 2013 and the link between the presence of the observed 1014F $k d r$ mutation and the resistance phenotype was not established. Another notable study followed increased frequency of 1014F $k d r$ in $A n$. coluzzii following the nationwide mass distribution of bed nets in Niger [7]. However, pyrethroids/DDT resistance profiling was not conducted on the Anopheles used in the study prior to genotyping.

To facilitate planning and implementation of evidencebased malaria control in Niger/the Sahel, the role of a major malaria vector $A n$. coluzzii, in malaria transmission in Niger, and its resistance status to the various insecticides in use for public health control was characterized. The underlying molecular mechanisms driving the resistance in the field was also investigated.

\section{Methods \\ Study site and collection}

Clearance for field work was sought and granted by the Niger Federal Ministry of Public Health (Authorization Number 003210/MSP/SG/DEP/DER). Indoor-resting mosquitoes were collected using battery-operated aspirators (John. W. Hock, Florida, USA) from six randomly selected houses, in the early morning hours (5:30 a.m.-6:30), in Takatsaba village, Tessaoua Department $\left(13.7559^{\circ} \mathrm{N}, 7.9865^{\circ} \mathrm{E}\right)$, Maradi State, Niger (Fig. 1). Collection was conducted every morning for 3 days (12-14 of September 2017). Takatsaba is a farming community, which banked the Goulbi River, allowing extended cultivation of rice and vegetables. Blood fed females were maintained on $10 \%$ sugar at $25{ }^{\circ} \mathrm{C} \pm 2$ and $70-75 \%$ relative humidity until fully gravid. 316 blood fed An. coluzzii females were individually transferred into $1.5 \mathrm{ml}$ tubes and forced to lay eggs, using established protocol [13]. The $\mathrm{F}_{0}$ parents identified as belonging to $A n$. gambiae complex using morphological keys [14] and confirmed as An. coluzzii using the SINE200-PCR [15] were selected for egg laying. Male Anopheles and Culex mosquitoes were discarded. Egg batches were transferred into paper cups for hatching in the insectary at Bayero University, Kano, Nigeria. Hatched eggs were pooled into larvae bowls and supplemented with Tetramin ${ }^{\mathrm{TM}}$ baby fish food. 2- to 4-days old $F_{1}$ female adults that emerged were randomly mixed in cages and used for insecticide bioassays.

To establish indoor resting densities of the Anopheles, infection with Plasmodium and source of blood meal, pyrethrum spray collection (PSC) was also conducted on the 4th day (15 of September 2017), following protocols as advised by the WHO [16]. PSC was carried out in 8 randomly selected houses in early morning hours (6:00 a.m.-7:00 a.m.). Mosquitoes were sorted according to morphological keys as either belonging to An. gambiae complex [14] or as Culex species and stored individually in PCR strips with silica gel.

\section{Anopheles species identification}

Genomic DNA was extracted from all the female Anopheles mosquitoes which successfully laid eggs using the Livak protocol [17]. Species identification to the 


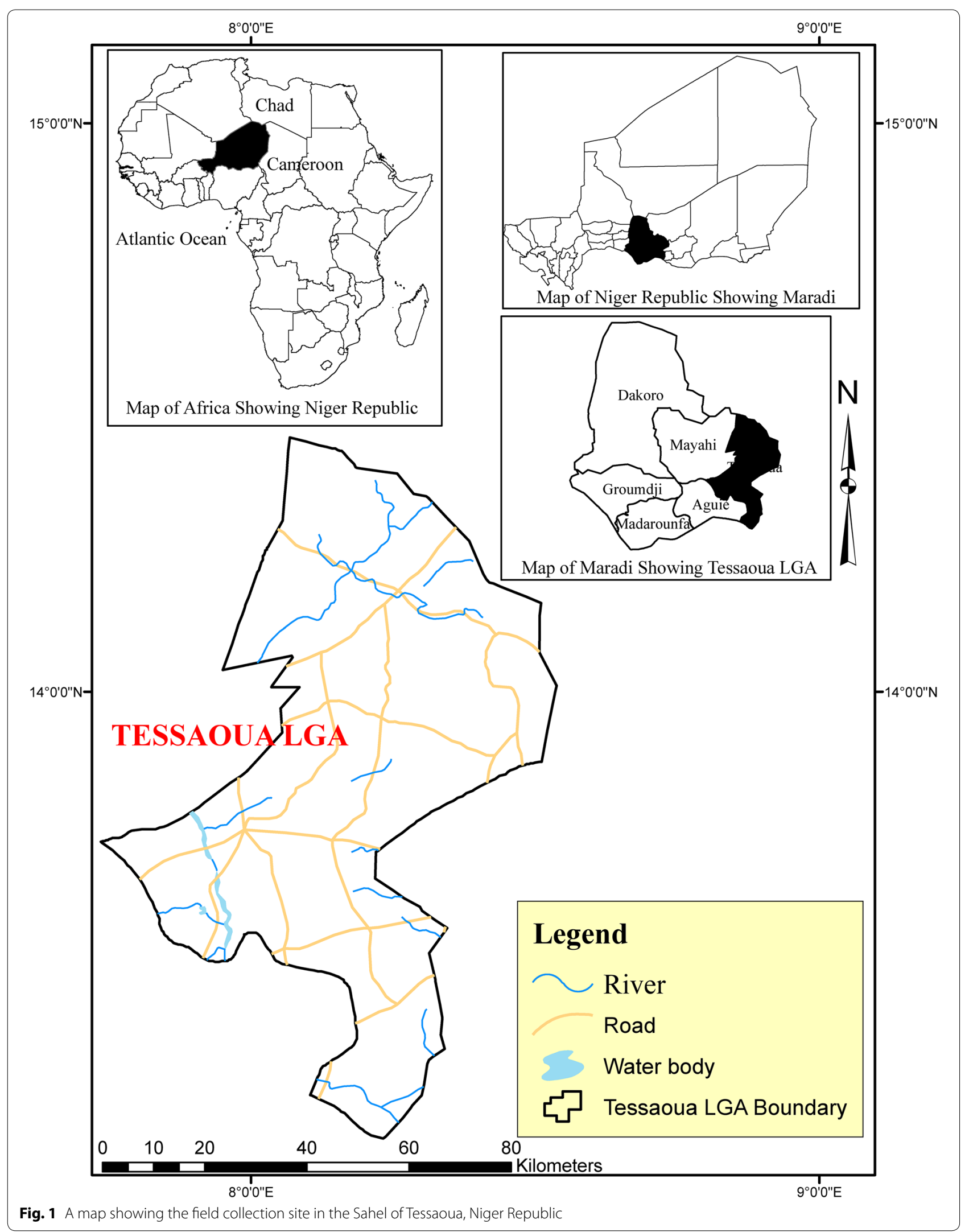


molecular level was carried out using the SINE200 PCR [15]. All female An. gambiae sensu lato collected from PSC were dissected to separate heads/thoraces from the abdomens. DNA was extracted from these heads/thoraces and species identification carried out as described above. These DNA samples were transported to the Liverpool School of Tropical Medicine (LSTM), UK, under the DEFRA license (PATH/125/2012,) for downstream molecular analyses.

\section{Estimation of transmission parameters Indoor resting density and human blood index estimation} The Anopheles indoor resting density (IRD) was calculated from the number of female Anopheles caught using the PSC in relation to the number of houses sampled as advised by the WHO $[16,18]$.

DNA was extracted from the abdomen of all blood fed female Anopheles as advised in the WHO guidelines [16]. This was done using the DNeasy Blood and Tissue Kit (QIAGEN, Hilden, Germany) according to manufacturer's protocol. The human blood index was established from the proportion of blood fed Anopheles that have fed on humans relative to the total number of blood fed Anopheles caught, following a cocktail PCR of Kent and Norris et al. [19].

\section{Sporozoite infection rate}

DNA from 93 female An. coluzzii collected using PSC were used to detect sporozoite using a TaqMan genotyping assay [20]. Mosquitoes were stored immediately after collection in a silica gel and DNA extracted within 7 days. To minimize blood contamination heads/thoraces were separated from abdomens at the anterior end of the abdomen [21]. Real-time PCR MX 3005 (Agilent, Santa Clara, USA) was used for the amplification. $1 \mu \mathrm{l}$ of gDNA extracted from each head/thorax was used, with an initial denaturation at $95{ }^{\circ} \mathrm{C}$ for $10 \mathrm{~min}$, followed by 40 cycles each of $15 \mathrm{~s}$ at $95^{\circ} \mathrm{C}$ and $1 \mathrm{~min}$ at $60^{\circ} \mathrm{C}$. Primers described by Bass [20] were used together with two probes labelled with fluorophores, FAM to detect Plasmodium falciparum, and HEX to detect combination of Plasmodium ovale, Plasmodium vivax and Plasmodium malariae. Positive controls (known FAM + and $\mathrm{OVM}+)$ were used, in addition to a negative control $(1 \mu \mathrm{l}$ of $\left.\mathrm{ddH}_{2} \mathrm{O}\right)$. TaqMan assay results were validated using a nested PCR [22]. This was carried out using all the positive TaqMan samples. Sporozoite rate was calculated as percentage of mosquitoes with sporozoites relative to the total number of the females examined [16].

\section{Tests for insecticides susceptibility}

Insecticide susceptibility bioassays were performed according to the WHO protocol [23] with the insecticides from four major public health classes. Four replicates of $F_{1}$ (20-25, 2-4 days old females) per tube were used for each insecticide, alongside 25 unexposed females (control). To confirm the efficacy of the papers, the fully susceptible An. coluzzii (Ngoussou colony) [24] was tested first or simultaneously with the experimental populations. Eight (8) insecticides were tested, including: (i) the type I pyrethroid: permethrin $(0.75 \%)$; (ii) the type II pyrethroids: deltamethrin $(0.05 \%)$ and $\alpha$-cypermethrin $(0.05 \%)$; (iii) the organochlorine: DDT (4\%); (iv) the carbamates: bendiocarb $(0.1 \%)$ and propoxur $(0.1 \%)$; and $(\mathrm{v})$ the organophosphates: malathion $(5 \%)$ and pirimiphosmethyl $(0.25 \%)$. Knockdown rates were recorded for permethrin, deltamethrin and DDT during the exposure, at intervals of $15 \mathrm{~min}, 30 \mathrm{~min}, 45 \mathrm{~min}$ and $1 \mathrm{~h}$. After $1 \mathrm{~h}$ exposure mosquitoes were transferred to holding tubes and supplied with $10 \%$ sugar. Mortality was recorded $24 \mathrm{~h}$ after exposure. Populations were considered susceptible to an insecticide where mortality was $>98 \%$, suspected to be resistant (moderately resistant) where mortality is between 90 and $98 \%$, and resistant where mortality was found to be $<90 \%$ [25].

\section{Estimation of resistance intensity with time-course bioassays}

To establish the strength of pyrethroid resistance with time, additional bioassays were performed with $0.05 \%$ deltamethrin. 20-25 $\mathrm{F}_{1}$ females in 4 replicates were exposed in time-course bioassays varying exposure times from 60 to $300 \mathrm{~min}$. Protocols were as described above in conventional bioassays, except for variation in time. For the fully susceptible Ngoussou $\mathrm{LT}_{50}$ have been established previously with discriminating concentration of deltamethrin in time-course bioassays spanning 2.5 to 60 min (Ibrahim et al., In Press, http://www.natur e.com/articles/s41598-019-43634-4). Resistance intensity was established by comparing the $\mathrm{LT}_{50}$ calculated for the Takatsaba An. coluzzii to that of the Ngoussou colony.

\section{Synergist bioassays}

To establish the potential role of detoxification enzyme systems in the observed resistance, synergist bioassays were conducted with 2-4 days old $\mathrm{F}_{1}$ females, with $4 \%$ piperonyl butoxide (PBO: an inhibitor of CYP450s [26]) against deltamethrin, and $8 \%$ diethyl maleate (DEM: an inhibitor glutathione S-transferases [27]). Initially, 20-25 females were pre-exposed to a synergist for $1 \mathrm{~h}$ and then transferred to tubes containing deltamethrin for $1 \mathrm{~h} \mathrm{[25].}$ Mosquitoes were treated as in the bioassays described above and mortalities scored after $24 \mathrm{~h}$. Two controls were used: (i) 25 females exposed to only control paper (with neither a synergist, nor any insecticide); and (ii) 25 females each exposed to either PBO or DEM only. 


\section{Polymorphism analysis of the voltage-gated sodium channel \\ Genotyping of L 1014F and L 1014S kdr mutations}

To assess the frequency and relation between $k d r$ genotypes and pyrethroid resistance phenotype, 55 survivors from permethrin exposure and the 19 dead females were genotyped for the presence of $1014 \mathrm{~F}$ and $1014 \mathrm{~S} k d r$ mutations. Genotyping was carried out using TaqMan real-time PCR thermocycler (Agilent Mx3005) following the protocols established by Bass and colleagues [28, 29]. $5 \mu$ l of Sensimix (Bioline), $0.25 \mu$ l of $40 \times$ Probe Mix coupled to allelic-specific primers, $4.25 \mu \mathrm{l}$ of $\mathrm{dH}_{2} \mathrm{O}$, and $1 \mu \mathrm{l}$ of genomic DNA were constituted in a total volume of $10 \mu \mathrm{l}$. Thermocycling conditions were initial $10 \mathrm{~min}$ at $95{ }^{\circ} \mathrm{C}$, followed by 40 cycles each of $92{ }^{\circ} \mathrm{C}$ for $15 \mathrm{~s}$, and $60{ }^{\circ} \mathrm{C}$ for $1 \mathrm{~min}$. Two probes labelled with fluorochromes FAM and HEX were used to detect the mutant alleles and the wild type susceptible alleles, respectively. Genotypes were scored from scatter plots of results produced by the Mx3005 v4.10 software. Three positive samples of known genotypes: (i) homozygote resistant for 1014F or $1014 \mathrm{~S} k d r$; (ii) heterozygote for $1014 \mathrm{~F}$ or $1014 \mathrm{~S} k d r$; and (iii) susceptible L1014 were added as positive controls for each of the two experiments. $1 \mu \mathrm{l}$ of $\mathrm{ddH}_{2} \mathrm{O}$ was added to the negative control well. The correlation between the $k d r$ genotypes and permethrin resistance phenotype was calculated by estimating the odds ratio (OR) and the statistical significance based on Fisher's exact probability test.

\section{Assessment of genetic diversity in the kdr locus of the voltage-gated sodium channel (VGSC)}

To assess the strength of selection pressure acting on the mosquito population in relation to insecticide resistance, the genetic diversity of a fragment spanning exon20 of the IIS6 of the VGSC (starting from intron-1 of the IIS6 to intron-2) was amplified from 8 each of the permethrin-alive and dead females. DNA was extracted as described above and amplification carried out with primer pairs: $k d r C L-F\left(5^{\prime}\right.$-AAATGTCTCGCCCAAATC AG-3') and $k d r C L-R$ (5'-GCACCTGCAAAACAATGT CA-3') described by Pinto [30]. To $12.5 \mu \mathrm{l}$ of the $2 \times$ AccuStartII PCR SuperMix (QuantaBio, Beverly, Massachusetts) containing optimized concentrations of $\mathrm{MgCl}_{2}$ and dNTP mixes, $0.2 \mu \mathrm{M}$ each of the forward and reverse primer was added, followed by $1 \mu \mathrm{l}$ cDNA. $10.5 \mu \mathrm{l}$ of $\mathrm{dH}_{2} \mathrm{O}$ was added to produce a total volume of $25 \mu \mathrm{l}$. Amplification was carried out using the following conditions: initial denaturation of one cycle at $94{ }^{\circ} \mathrm{C}$ for $3 \mathrm{~min}$; followed by 35 cycles each of $94{ }^{\circ} \mathrm{C}$ for $30 \mathrm{~s}$ (denaturation), $60{ }^{\circ} \mathrm{C}$ for $30 \mathrm{~s}$ (annealing), and extension at $72{ }^{\circ} \mathrm{C}$ for $1 \mathrm{~min}$; and one cycle at $72{ }^{\circ} \mathrm{C}$ for $5 \mathrm{~min}$ (final elongation). PCR products were cleaned individually with
QIAquick ${ }^{\circledR}$ PCR Purification Kit (QIAGEN, Hilden, Germany) and sequenced on both strands using the above primers.

Polymorphisms were detected through manual examination of sequence traces using BioEdit version 7.2.3.0 [31] and CLC sequence viewer (QIAGEN, Hilden, Germany), whereas haplotype reconstruction and analyses of genetic parameters of polymorphism were done using the DnaSP 5.10 [32]. Different sequences were compared by constructing a phylogenetic maximum likelihood tree using MEGA 6.0 [33]. To estimate genealogies between sequences of the alive and the dead mosquitoes, haplotype network was created with the TCS (http://darwi n.uvigo.es/software/tcs.html) and tscBU [34]. All DNA sequences from the alive and dead females were submitted to the GenBank (MK440265-440280).

\section{Statistical analyses}

To calculate the odds ratio the epiR package of $\mathrm{R}$ version $\quad 3.5 .0 \quad$ (https://cran.r-project.org/bin/windows/ base/) was utilized. Estimation of $\mathrm{LT}_{50}$ was also carried out with probit analyses (glm with MASS package of R). Result of mortalities from synergist-deltamethrin exposure was compared with that obtained from exposure to deltamethrin alone using a two-tailed Chi Square test of independence as implemented in GraphPad Prism 7.02 (GraphPad Inc., La Jolla, CA, USA) and correlation between $k d r$ genotype and permethrin resistance phenotype determined using Fisher's exact test.

\section{Results}

\section{Mosquito species composition}

Out of the 484 mosquitoes caught resting indoor, 445 were Anopheles [51 0,394 9 (316 blood fed and 78 unfed)], 39 were of Culex species (27\% blood fed and 12 ${ }^{\text {)}}$. All the Anopheles were established to be $A n$. coluzzii. From the 316 female An. coluzzii transferred into $1.5 \mathrm{ml}$ only 147 (46.5\%) laid eggs successfully, due to high mortality experienced while travelling to insectary at Kano, Nigeria. A total of 117 mosquitoes were caught from PSC, 106 of them An. coluzzii [90, 970 (95 blood fed and 2 unfed)], and 11 were of Culex species (8q blood fed and $3 \sigma^{\top}$ ).

\section{Malaria transmission parameters Indoor-resting density and human blood index}

Indoor resting density of the female $A n$. coluzzii was calculated from the number of females collected as 12.125 females per house. DNA extracted from 95 blood fed females collected using PSC was analysed for blood source. 91 of the abdomens contained human blood, 2 abdomens contained cow blood, and the rest failed. The human blood index was found to be very high, calculated 
as 0.957 ( $\sim 96 \%$ of the foraging female An. coluzzii have fed on humans).

\section{Estimation of sporozoites infection rate}

93 DNA-extracted heads/thoraces from PSC-caught An. coluzzii were used to establish infection with Plasmodium. 13 mosquitoes were infected with Plasmodium. 12 heads/thoraces contained $P$. falciparum $(\mathrm{F}+=12)$, corresponding to a sporozoite rate of $12.9 \%$, and a single sample was also found infected with $P$. ovale/P. vivax/P. malariae (OVM). Nested PCR confirmed this parasite as P. $\operatorname{vivax}(\mathrm{V}+=1.0)$.

\section{WHO insecticide susceptibility bioassays}

The fully susceptible Ngoussou exhibited 100\% mortalities against all insecticides tested. Bioassays with Takatsaba females revealed high resistance to pyrethroids and
DDT with no knockdown at all for permethrin, deltamethrin and DDT in the first 30 min of exposure (Fig. 2a). At 45 min only a single mosquito was knocked down from permethrin exposure; and 2 from DDT. For DDT knockdown rate increased to only 5 individuals at $1 \mathrm{~h}$, while only a single individual was knocked down for permethrin and deltamethrin, respectively at $1 \mathrm{~h}$.

For the pyrethroids, mortalities of only 19\% (95\% CI 18-20), 17\% (CI 16-18) and 14\% (CI 9-19) were obtained at $24 \mathrm{~h}$ post-exposure, with permethrin, deltamethrin and $\alpha$-cypermethrin, respectively (Fig. 2b). The same pattern was observed with DDT with only $18 \%$ (CI 16-19) of females dead at $24 \mathrm{~h}$.

In contrast, the mosquitoes were more susceptible to the non-pyrethroid insecticides. Mortalities of more than $90 \%$ were observed for the carbamates bendiocarb and propoxur, while full susceptibility was seen with the
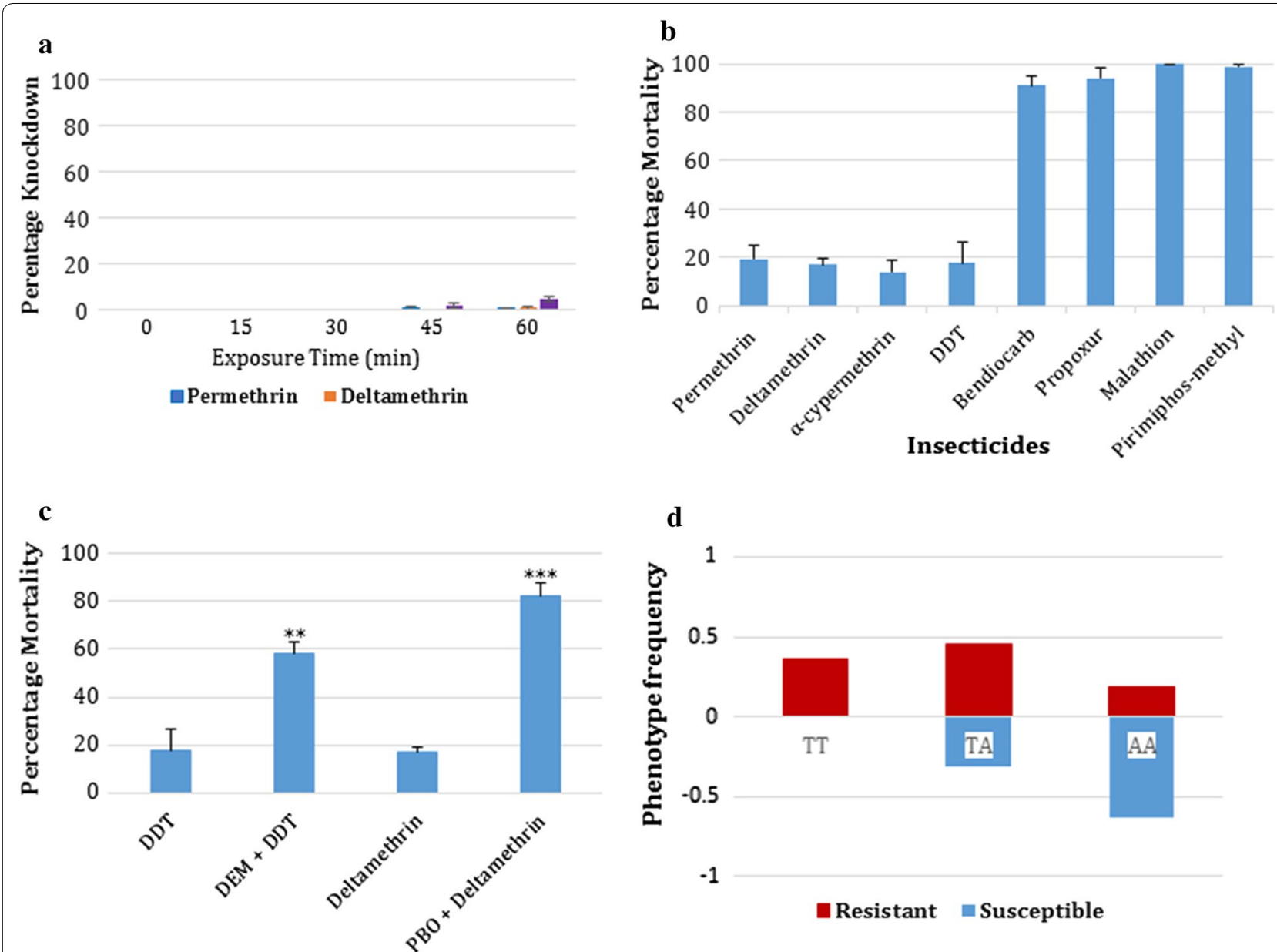

Fig. 2 Resistance profiles of $F_{1}$ Takatsaba Anopheles coluzzii females. a Knockdown resistance profile with permethrin, deltamethrin and DDT; b WHO bioassays with insecticides from different classes. c Effect of pre-exposure to synergists PBO against deltamethrin, and with DEM against DDT. Results shown are average of percentage mortalities from 4 replicates each \pm SEM. No mortality was obtained in control females and positive control females exposed to DEM and PBO alone; ** and ${ }^{* * *}$ significantly different from exposure to DDT and deltamethrin alone, at $\mathrm{p}<0.001$ and $p<0.0001$. d Correlation between the kdr genotype and resistance phenotypes in alive and dead Takatsaba An. coluzzii 
organophosphates, malathion and pirimiphos-methyl (Fig. 2b).

\section{Estimation of resistance intensity}

To establish levels of resistance WHO tube bioassays were conducted with deltamethrin varying exposure times from 60 to $300 \mathrm{~min}$. The $\mathrm{LT}_{50}$ of Takatsaba $A n$. coluzzii population was estimated as $156.65 \mathrm{~min}$ ( $95 \%$ CI 144.10-169.21, Fiducial). The $\mathrm{LT}_{50}$ for the Ngoussou has been estimated as $3.320 \mathrm{~min}$ (CI 2.67-3.97) from a previous study (Ibrahim et al. In Press, http://www.natur e.com/articles/s41598-019-43634-4). The resistance ratio for Takatsaba females compared with the Ngoussou colony was thus calculated as 47.18 .

\section{Investigating the potential role of metabolic resistance using synergist bioassays}

Pre-exposure to the synergists recovered susceptibility to both deltamethrin and DDT, suggesting the possible role of cytochrome P450s and glutathione S-transferases respectively, in the resistance to the above insecticides. A high recovery of susceptibility was obtained following pre-exposure to $\mathrm{PBO}$ with mortality increasing $\sim 5$ times, from $17 \%$ seen in the conventional bioassay to $82 \%$ (CI 76-88) (Fig. 2c). Two-tailed test of independence indicated that the association between the mortality and PBO pre-exposure is highly significant $\left(\chi^{2}=84.51, \mathrm{df}=1\right.$, $\mathrm{p}<0.0001)$. DDT mortality increased also following preexposure to DEM, from $18 \%$ in the conventional bioassay to $58 \%$ (CI $\left.52-65, x^{2}=33.96, d f=1, p<0.001\right)$. No mortality was observed in all controls.

\section{Polymorphism analysis of the voltage-gated sodium channel \\ Investigation of the role of $1014 \mathrm{~F}$ and $1014 \mathrm{~S}$ kdr mutations on pyrethroid resistance}

To determine correlation between the resistance phenotype and $k d r$ genotype, 55 permethrin-resistant and 19 dead $\mathrm{F}_{1}$ females were genotyped for $1014 \mathrm{~F}$ and $1014 \mathrm{~S} k d r$ mutations. High frequency of 1014F $k d r$ mutation was obtained with 20 females (36.36\%) homozygote resistant (T/T) for 1014F $k d r$ mutation (Table 1), and 25 females
(45.45\%) heterozygote resistant (T/A). Only 10 females (18.18\%) harbour the homozygote susceptible allele (A/A). The 1014F $k d r$ genotype frequency was established in the resistant females as $\sim 0.82$, and for all alive and dead females as $70.3 \%$. Not surprisingly, out of the 19 permethrin-susceptible females successfully genotyped, $12(63.15 \%)$ were homozygote susceptible at the 1014 codon (A/A); none were homozygote resistant; and $7(36.84 \%)$ were heterozygotes (T/A). The $k d r$ genotype frequency in the susceptible mosquitoes was established as 0.37 . The $1014 \mathrm{~S} k d r$ mutation was not detected in all mosquitoes genotyped.

A significant difference in genotype distribution was observed between the permethrin-resistant and susceptible individuals (Table 1, Fig. 2d), with $63 \%$ of susceptible females homozygote susceptible for 1014F $k d r$, while only $18 \%$ of the resistant females harbours the susceptible allele. A significant correlation was observed between permethrin resistance and presence of $1014 \mathrm{~F}$ mutation [odds ratio of $7.71\left(95 \%\right.$ CI 2.43-14.53, $\chi^{2}=13.67$, $\mathrm{p}=0.001$ )] (Table 1) when comparing the frequency of the resistance (homozygote resistant, RR + heterozygote resistant, RS) with susceptible allele (SS), in all females genotyped. However, stronger association was observed in the RR females in relation to the SS females [OR of 22 (CI 2.48-95.27, $\chi^{2}=11.67, \mathrm{p}=0.001$ )] compared to above and to the association observed between the SS and the heterozygote females (RS) [OR of 4.29 (CI 1.31-14.03, $\left.\left.x^{2}=6.10, p=0.014\right)\right]$.

\section{Genetic diversity of the IIS6 fragment of the voltage-gated sodium channel}

A 545 bp sequence was successfully retrieved from sequencing data and analysed for genetic diversity. This fragment spanning exon-20 of the IIS6 of the VGSC from 8 each of alive and dead females revealed a higher heterogeneity within the alive mosquitoes compared to the dead ones. Six sequences each from the alive and dead formed two predominant clusters: Hap_1 and Hap_5 respectively (Fig. $3 \mathrm{a}-\mathrm{c}$ ) corresponding to the resistant allele 1014F (Hap_1) and to the susceptible allele L1014 (Hap_5). However, sequences from two dead mosquitoes

Table 1 Correlation between the 1014F allele frequency and permethrin resistance phenotype in Takatsaba Anopheles coluzzii populations

\begin{tabular}{|c|c|c|c|c|c|c|c|c|c|}
\hline \multirow[t]{2}{*}{ Population } & \multirow[t]{2}{*}{ Phenotype } & \multirow[t]{2}{*}{$\mathbf{n}$} & \multicolumn{3}{|c|}{ L1014F alleles } & \multirow{2}{*}{$\begin{array}{l}\% k d r \\
\text { frequency } \\
\text { (RR+RS) }\end{array}$} & \multirow[t]{2}{*}{$k d r$ allele } & \multirow[t]{2}{*}{ Odds ratio (RR + RS vs SS) } & \multirow{2}{*}{$\begin{array}{l}F \\
p \text { value }\end{array}$} \\
\hline & & & TTT (RR) & TTT/A (RS) & TTA (SS) & & & & \\
\hline \multirow[t]{2}{*}{ Takatsabą } & Alive & 55 & $20(36.36 \%)$ & $25(45.45 \%)$ & $10(18.18 \%)$ & $45(81.81 \%)$ & 0.82 & $7.71(2.43-14.53)$ & $(0.001)$ \\
\hline & Dead & 19 & $0(0 \%)$ & $7(36.84 \%)$ & $12(63.15 \%)$ & $7(36.84 \%)$ & 0.37 & & \\
\hline Total & & 74 & $20(27.02 \%)$ & 32 (43.24\%) & $22(29.73 \%)$ & $52(70.27 \%)$ & & & \\
\hline
\end{tabular}

n, number of successfully genotyped individuals. Numbers in brackets represent percentage frequency. TTT: homozygote resistant alleles (RR); TTT/A: heterozygote resistant; and TTA: homozygote susceptible. F, Fisher's exact test 


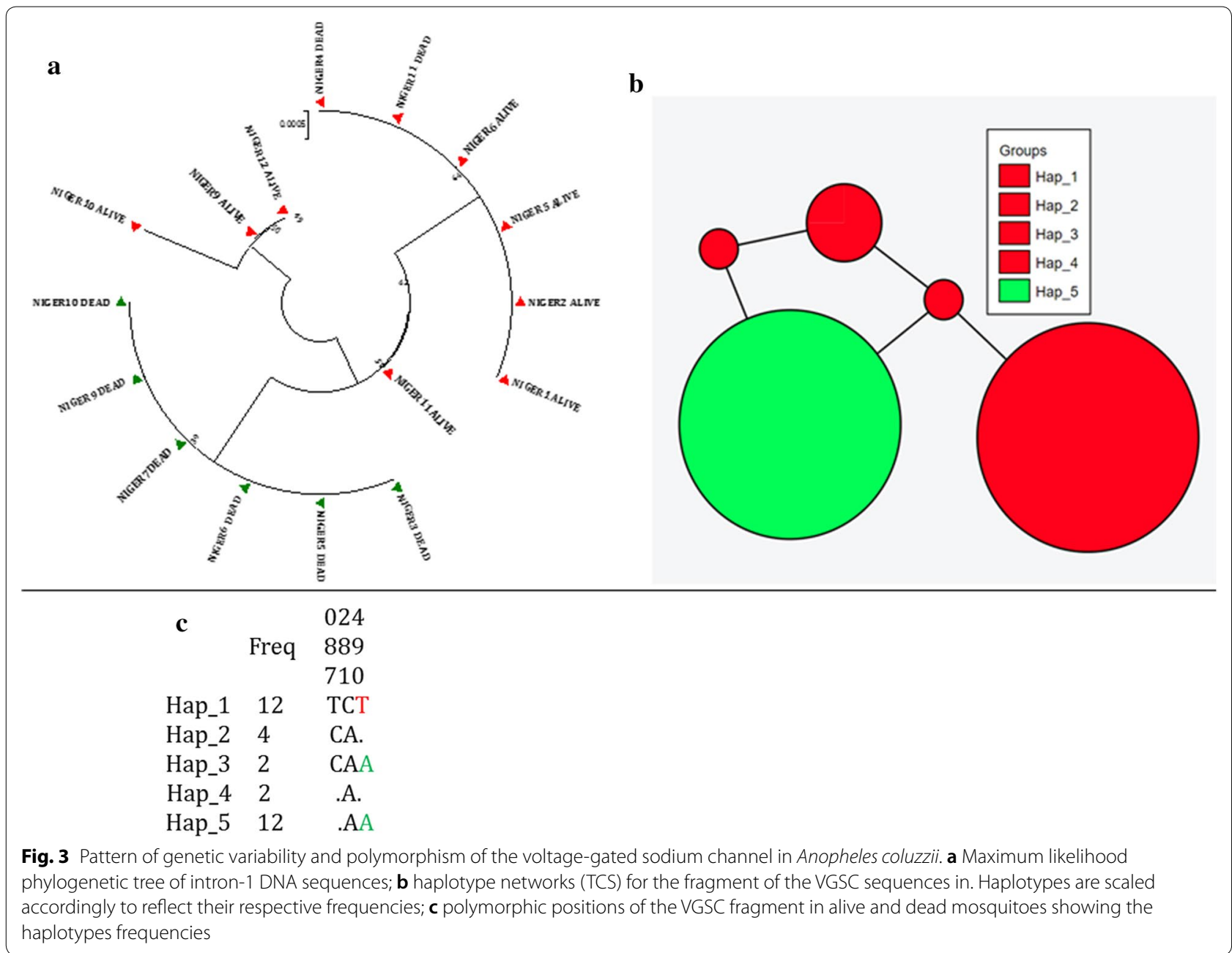

cluster into Hap_1, resulting in 2 haplotypes for the dead mosquitoes, and lower haplotype diversity (Fig. 3a-c, Table 2), compared to the alive mosquitoes. Indeed, the alive mosquitoes contributed 4 haplotypes out of 5 with a diversity on average twice that of the dead mosquitoes, suggesting a potential segregation event taking place within intron-1 of the VGSC in the alive mosquitoes. Except for the 1014 codon, no other polymorphism (synonymous or nonsynonymous) was seen in the exon-20 in both alive and dead mosquitoes. Both the other two mutations were seen in intron-1, and none in intron-2.

\section{Discussion}

Failure to make significant progress in malaria control in high burden countries like Niger Republic is jeopardising the WHO's Global Technical Strategy for Malaria 2016-2030. Control efforts in Niger, like other Sahelian zone countries are not enough, due in part to lack of

Table 2 Summary statistics for polymorphism of the fragment of voltage-gated sodium channel haplotypes between alive and dead mosquitoes

\begin{tabular}{llllllllll}
\hline Phenotype & $\mathbf{N}$ & $\mathbf{S}$ & $\mathbf{h}$ & $\mathbf{H}_{\mathbf{d}}$ & Syn & Nonsyn & $\boldsymbol{\pi}(\mathbf{k})$ & $\mathbf{D}$ (Tajima) & $\mathbf{D}^{*}$ (Fu and Li) \\
\hline Alive & 8 & 3 & 4 & 0.70 & 0 & 1 & $0.00230(1.266)$ & $1.16^{\mathrm{ns}}$ & $1.04^{\mathrm{ns}}$ \\
Dead & 8 & 2 & 2 & 0.40 & 0 & 1 & $0.01470(0.800)$ & $0.84^{\mathrm{ns}}$ & $0.91^{\mathrm{ns}}$ \\
All & 16 & 3 & 5 & 0.72 & 0 & 1 & $0.0024(1.306)$ & $0.94^{\mathrm{ns}}$ & $1.36^{\mathrm{ns}}$
\end{tabular}

$\mathrm{N}$, number of sequences $(n) ; \mathrm{S}$, number of polymorphic sites; $h$, haplotype; $\mathrm{H}_{d}$, haplotype diversity; Syn, synonymous mutations; Nonsyn, non-synonymous mutations; $\pi$, nucleotide diversity ( $k=$ mean number of nucleotide differences); Tajima's D and Fu and Li's D statistics, ns, not significant 
reliable surveillance data to guide decision making by the national malaria control programmes. To facilitate evidence-based malaria control approaches and resistance management in Niger, we established the role of the major malaria vector $A n$. coluzzii in malaria transmission in an area of stable transmission. Insecticide resistance profile of this malaria vector was established and the possible molecular mechanisms driving the resistance in the field investigated. However, conclusions of this study are drawn from a single collection, at one site. To capture the actual level/variation in Plasmodium infection and pattern of insecticide resistance in this malaria vector across southern Niger there is a need to carry out further collection at different time points, and several sites spanning the Sahel of the country.

Over the years, An. coluzzii has progressively become the DVS in the Sahel zone of Niger compared to the Anopheles arabiensis which is hard to come by, and $A n$. gambiae sensu stricto, which has virtually disappeared in this region $[9,11]$. Indeed, in recent years this species has been reported more than the other sibling species in various studies from the Sudan/Sahel of the neighbouring countries (e.g. in northern Nigeria [35], in northern Chad [36] and in Mali [37]), suggesting that this malaria vector has probably adapted well in the Sahel regions.

Also, Plasmodium transmission competence of An. coluzzii has increased in Niger with time. Previous studies have reported sporozoite rate of no more than $3 \%$ in various sites across Niger $[10,11]$. The finding of $P$. falciparum sporozoite rate of $\sim 13 \%$ in this study suggests that this vector is sustaining a high level of malaria transmission despite ongoing control efforts. This coupled with the high anthropophagic index of more than 95\% suggests that this species has become a very efficient transmitter of malaria parasite in the Sahel and must be targeted to reduce malaria burden in this region. However, the high infection observed might also be because some of the females had recently taken blood from infected individuals.

The fact that mortalities of less than a quarter was recorded for the pyrethroids and DDT suggests that the resistance to these nerve agents have escalated in An. coluzzii from southern Niger. In comparison to the findings of Soumaila and colleagues [9] at Tessaoaua, resistance towards permethrin and $\alpha$-cypermethrin has doubled and quadrupled, respectively in a few years. This is possibly due to increased selection pressure from second wave of distribution of PermaNet2.0 LLIN between 2014 and 2017. Also, in contrast to the previous observation of full susceptibility towards carbamates [9] marginal resistance was observed, especially towards bendiocarb. The level of bendiocarb resistance resembles recent observation in the Sahel of Nigeria where An. coluzzii populations exhibited comparable moderate resistance (Ibrahim et al. In Press, http://www.nature.com/articles/s41598-019-43634-4). However, as previously reported the mosquitoes were fully susceptible to the organophosphates. The high pyrethroid resistance observed in the Tessaoaua populations is evident in its high $\mathrm{LT}_{50}$ with deltamethrin, which is on average higher than the values described previously for resistant populations of An. gambiae from the Tororo (Uganda) and Tiefora (Burkina Faso) [38].

The high recovery of resistance on pre-exposure to $\mathrm{PBO}$ and DEM points to the potential role of $\mathrm{P} 450$ monooxygenases and glutathione $\mathrm{S}$-transferases, respectively in the resistance. Previous studies have reported the use of synergists to investigate contribution of metabolic resistance in various An. gambiae populations [27, 39].

A previous study has reported the $1014 \mathrm{~F} k d r$ frequency of 56\% in An. coluzzii from Tessaoua [9]. This frequency has increased to $\sim 82 \%$ in 5 years, like the observations in the neighbouring Sahel region of Nigeria [35]. The low haplotype and nucleotide diversity seen in the Niger populations agrees with the previous observation that Western African sites variation at the intron-1 was lowest compared to other regions of Africa [30]. The low genetic diversity (highlighted by the very low number of haplotypes) suggests a restricted polymorphism of the VGSC, in link with the near fixation of the 1014F allele in this population. These findings are consistent with previous studies reported as reported in West [35] and Central Africa [40].

\section{Conclusion}

In a few decades $A n$. coluzzii has adapted to the dry conditions of the Sahel region to become the major malaria vector (e.g. in Niger). Pyrethroid resistance in this species has escalated and will pose a threat to the malaria control/elimination agenda. Of significant importance also is the carbamate resistance observed in the field which could confound feature control measures using carbamate-based the indoor residual spraying. It is necessary to continue monitoring the spacio-temporal pattern of this resistance in the south of Niger and to carry out detailed studies on the contribution of metabolic resistance mechanisms operating in the field populations of this species from Niger. This will help revamp the effort towards malaria control in Niger/Sahelian region in general. 


\section{Acknowledgements}

This work was supported by the Wellcome Trust International Training Fellowship in Public Health and Tropical Medicine (WT201918/Z/16/Z) to SSI.

\section{Authors' contributions}

Conceived and designed by SSI with contributions from CSW. SSI, RL, and IM carried out the field collection of mosquitoes. SSI, MMM, MOK and HI conducted the insecticide bioassays. SSI, HI and MMM carried out molecular analyses. SSI carried out data analyses and wrote the manuscript with contribution from CSW and all authors. All authors read and approved the final manuscript.

\section{Funding}

A Wellcome Trust International Training Fellowship in Public Health and Tropical Medicine (WT201918/Z/16/Z) to SSI supported this work.

\section{Availability of data}

DNA sequences reported in this paper were deposited at GenBank (Accession Number: MK440265-440280).

\section{Ethics approval and consent to participate}

Clearance for field work and ethical approval was granted by the Niger Federal Ministry of Public Health (Authorization Number 003210/MSP/SG/DEP/DER). This approval letter was presented to the Comprehensive Health Centre at Tessaoua, which granted permission to proceed to Takatsaba village. Prior to collection, a meeting was held with the local chief and the village people to explain the aim of the study and its benefits. For individuals who agreed to indoor collection in their houses a written and signed informed consent was obtained. All communication was carried out in the local language which is Hausa.

\section{Consent for publication}

Not applicable.

\section{Competing interests}

The authors declare that they have no competing interests.

\section{Author details}

${ }^{1}$ Vector Biology Department, Liverpool School of Tropical Medicine (LSTM) Pembroke Place L3 5QA, UK. ${ }^{2}$ Department of Biochemistry, Bayero University, PMB 3011, Kano, Nigeria. ${ }^{3}$ Centre de Recherche Médicale et Sanitaire (CERMES), Institut Pasteur International Network, 634 Bd de la Nation, BP 10887, Niamey, Niger. ${ }^{4}$ LSTM Research Unit, Centre for Research in Infectious Diseases (CRID), P.O. Box 13591, Yaoundé, Cameroon.

\section{Received: 4 February 2019 Accepted: 15 May 2019}

Published online: 24 May 2019

\section{References}

1. Alonso P, Noor AM. The global fight against malaria is at crossroads. Lancet. 2017:390:2532-4.

2. WHO. World malaria report. Geneva: World Health Organization; 2018

3. Bhatt S, Weiss D, Cameron E, Bisanzio D, Mappin B, Dalrymple U, et al. The effect of malaria control on Plasmodium falciparum in Africa between 2000 and 2015. Nature. 2015:526:207-11.

4. WHO. World malaria report. Geneva: World Health Organization; 2017.

5. WHO. Global technical strategy for malaria 2016-2030. Geneva: World Health Organization; 2015.

6. PMI. Niger Malaria Operational Plan FY 2017. Niamey; 2017.

7. Czeher C, Labbo R, Arzika I, Duchemin J-B. Evidence of increasing Leu-Phe knockdown resistance mutation in Anopheles gambiae from Niger following a nationwide long-lasting insecticide-treated nets implementation. Malar J. 2008;7:189.

8. Cairns M, Roca-Feltrer A, Garske T, Wilson AL, Diallo D, Milligan PJ, et al. Estimating the potential public health impact of seasonal malaria chemoprevention in African children. Nat Commun. 2012;3:881.

9. Soumaila H, Idrissa M, Akgobeto M, Habi G, Jackou H, Sabiti I, et al. Multiple mechanisms of resistance to pyrethroids in Anopheles gambiae s.l. populations in Niger. Med Mal Infect. 2017;47:415-23.
10. Labbo R, Czeher C, Djibrila A, Arzika I, Jeanne I, Duchemin JB. Longitudinal follow-up of malaria transmission dynamics in two villages in a Sahelian area of Niger during a nationwide insecticide-treated bednet distribution programme. Med Vet Entomol. 2012;26:386-95.

11. Labbo R, Fandeur T, Jeanne I, Czeher C, Williams E, Arzika I, et al. Ecology of urban malaria vectors in Niamey, Republic of Niger. Malar J. 2016;15:314.

12. Loewenberg S. Niger welcomes largest bednet distribution in history. Lancet. 2006:367:1473.

13. Morgan JC, Irving H, Okedi LM, Steven A, Wondji CS. Pyrethroid resistance in an Anopheles funestus population from Uganda. PLoS One. 2010;5:7.

14. Gillies M, Coetzee M. A supplement to anophelinae of Africa south of Sahara (Afro-tropical region). South Africa Inst Med Res. 1987;55:1-143.

15. Santolamazza F, Mancini E, Simard F, Qi Y, Tu Z, Torre A. Insertion polymorphisms of SINE200 retrotransposons within speciation islands of Anopheles gambiae molecular forms. Malar J. 2008;7:163.

16. WHO. Malaria entomology and vector control. Guide for participants. Geneva, World Health Organization. 2013.

17. Livak KJ. Organization and mapping of a sequence on the Drosophila melanogaster $X$ and $Y$ chromosomes that is transcribed during spermatogenesis. Genetics. 1984;107:611-34.

18. WHO. A toolkit for integrated vector management in sub-Saharan Africa. Geneva: World Health Organization; 2016.

19. Kent RJ, Thuma PE, Mharakurwa S, Norris DE. Seasonality, blood feeding behavior, and transmission of Plasmodium falciparum by Anopheles arabiensis after an extended drought in southern Zambia. Am J Trop Med Hyg. 2007;76:267-74.

20. Bass C, Nikou D, Blagborough AM, Vontas J, Sinden RE, Williamson MS, et al. PCR-based detection of Plasmodium in Anopheles mosquitoes: a comparison of a new high-throughput assay with existing methods. Malar J. 2008:7:177.

21. Foley DH, Harrison G, Murphy JR, Dowler M, Rueda LM, Wilkerson RC. Mosquito bisection as a variable in estimates of PCR-derived malaria sporozoite rates. Malar J. 2012;11:145.

22. Snounou G, Viriyakosol S, Zhu XP, Jarra W, Pinheiro L, Rosario VE, et al. High sensitivity of detection of human malaria parasites by the use of nested polymerase chain reaction. Mol Biochem Parasitol. 1993;61:315-20.

23. WHO. Test procedures for insecticide resistance monitoring in malaria vector mosquitoes. Geneva: World Health Organization; 2013.

24. Mitchell SN, Stevenson BJ, Müller P, Wilding CS, Egyir-Yawson A, Field SG, et al. Identification and validation of a gene causing cross-resistance between insecticide classes in Anopheles gambiae from Ghana. Proc Nat Acad of Sci USA. 2012;109:6147-52.

25. WHO. Test procedures for insecticide resistance monitoring in malaria vector mosquitoes. Geneva: World Health Organization; 2016.

26. Feyereisen R. Insect P450 inhibitors and insecticides: challenges and opportunities. Pest Man Sci. 2015;71:793.

27. Chouaïbou M, Zivanovic GB, Knox TB, Jamet HP, Bonfoh B. Synergist bioassays: A simple method for initial metabolic resistance investigation of field Anopheles gambiae s.l. populations. Acta Trop. 2014;130:108-11.

28. Bass C, Nikou D, Donnelly MJ, Williamson MS, Ranson H, Ball A, et al. Detection of knockdown resistance (kdr) mutations in Anopheles gambiae: a comparison of two new high-throughput assays with existing methods. Malar J. 2007;6:111.

29. Bass C, Nikou D, Vontas J, Donnelly MJ, Williamson MS, Field LM. The vector population monitoring tool (VPMT): high-throughput DNA-based diagnostics for the monitoring of mosquito vector populations. Malar Res Treatm. 2010;1:1. https://doi.org/10.4061/2010/190434.

30. Pinto J, Lynd A, Vicente JL, Santolamazza F, Randle NP, Gentile G, et al. Multiple origins of knockdown resistance mutations in the Afrotropical mosquito vector Anopheles gambiae. PLoS ONE. 2007;2:11.

31. Hall TA. BioEdit: a user-friendly biological sequence alignment editor and analysis program for Windows 95/98/NT. Nucleic Acids Symp Ser. 1999;41:95-8.

32. Librado P, Rozas J. DnaSP v5: a software for comprehensive analysis of DNA polymorphism data. Bioinformatics. 2009;25:1451-2.

33. Tamura K, Stecher G, Peterson D, Filipski A, Kumar S. MEGA6: molecular evolutionary genetics analysis version 6.0. Mol Biol Evol. 2013;30:2725-9. 
34. Santos A, Cabezas MP, Tavares Al, Xavier R, Branco M. tcsBU: a tool to extend TCS network layout and visualization. Bioinformatics. 2016;32:627-8

35. Ibrahim SS, Manu YA, Tukur Z, Irving H, Wondji CS. High frequency of kdr L1014F is associated with pyrethroid resistance in Anopheles coluzzii in Sudan savannah of northern Nigeria. BMC Inf Dis. 2014;14:441.

36. Diarra A, Dabo A, Saye R, Coulibaly D, Guindo M, Sagara I, et al. Entomological and parasitological parameters of malaria transmission in Douguia, Chad. Med Sante Trop. 2017:27:253-9.

37. Krajacich BJ, Huestis DL, Dao A, Diallo M, Krishna A, Xu J, et al. Investigation of the seasonal microbiome of Anopheles coluzzii mosquitoes in Mali. PLoS One. 2018;13:3.

38. Bagi J, Grisales N, Corkill R, Morgan JC, N'Falé S, Brogdon WG, et al. When a discriminating dose assay is not enough: measuring the intensity of insecticide resistance in malaria vectors. Malar J. 2015;14:210.
39. Dadzie SK, Chabi J, Asafu-Adjaye A, Owusu-Akrofi O, Baffoe-Wilmot A, Malm K, et al. Evaluation of piperonyl butoxide in enhancing the efficacy of pyrethroid insecticides against resistant Anopheles gambiae s.l. in Ghana. Malar J. 2017;16:342.

40. Riveron JM, Watsenga F, Irving H, Irish SR, Wondji CS. High Plasmodium infection rate and reduced bed net efficacy in multiple insecticide-resistant malaria vectors in Kinshasa, Democratic Republic of Congo. J Inf Dis. 2017;217:320-8.

\section{Publisher's Note}

Springer Nature remains neutral with regard to jurisdictional claims in published maps and institutional affiliations.
Ready to submit your research? Choose BMC and benefit from:

- fast, convenient online submission

- thorough peer review by experienced researchers in your field

- rapid publication on acceptance

- support for research data, including large and complex data types

- gold Open Access which fosters wider collaboration and increased citations

- maximum visibility for your research: over $100 \mathrm{M}$ website views per year

At BMC, research is always in progress.

Learn more biomedcentral.com/submissions 